\title{
Metabolic engineering for the production of shikimic acid in an evolved Escherichia coli strain lacking the phosphoenolpyruvate: carbohydrate phosphotransferase system
}

\author{
Adelfo Escalante*1, Rocío Calderón', Araceli Valdivia', Ramón de Anda', Georgina Hernández', Octavio T Ramírez², \\ Guillermo Gosset ${ }^{1}$ and Francisco Bolívar ${ }^{1}$
}

\begin{abstract}
Background: Shikimic acid (SA) is utilized in the synthesis of oseltamivir-phosphate, an anti-influenza drug. In this work, metabolic engineering approaches were employed to produce SA in Escherichia coli strains derived from an evolved strain (PB12) lacking the phosphoenolpyruvate:carbohydrate phosphotransferase system (PTS-) but with capacity to grow on glucose. Derivatives of PB12 strain were constructed to determine the effects of inactivating aroK, aroL, pykF or pykA and the expression of plasmid-coded genes aroGfbr, $t k t A$, aroB and $\operatorname{aro} E$, on SA synthesis.

Results: Batch cultures were performed to evaluate the effects of genetic modifications on growth, glucose consumption, and aromatic intermediate production. All derivatives showed a two-phase growth behavior with initial high specific growth rate $(\mu)$ and specific glucose consumption rate (qs), but low level production of aromatic intermediates. During the second growth phase the $\mu$ decreased, whereas aromatic intermediate production reached its maximum. The double aroK-aroL- mutant expressing plasmid-coded genes (strain PB12.SA22) accumulated SA up to $7 \mathrm{~g} / \mathrm{L}$ with a yield of SA on glucose of $0.29 \mathrm{~mol} / \mathrm{mol}$ and a total aromatic compound yield (TACY) of $0.38 \mathrm{~mol} / \mathrm{mol}$. Single inactivation of pykF or pykA was performed in PB12.SA22 strain. Inactivation of pykF caused a decrease in $\mu, q 5$, SA production, and yield; whereas TACY increased by $33 \%(0.5 \mathrm{~mol} / \mathrm{mol})$.

Conclusions: The effect of increased availability of carbon metabolites, their channeling into the synthesis of aromatic intermediates, and disruption of the SA pathway on SA production was studied. Inactivation of both aroK and aroL, and transformation with plasmid-coded genes resulted in the accumulation of $S A$ up to $7 \mathrm{~g} / \mathrm{L}$ with a yield on glucose of $0.29 \mathrm{~mol} / \mathrm{mol}$ PB12.SA22, which represents the highest reported yield. The pykF and pykA genes were inactivated in strain PB12.SA22 to increase the production of aromatic compounds in the PTS- background. Results indicate differential roles of Pyk isoenzymes on growth and aromatic compound production. This study demonstrated for the first time the simultaneous inactivation of PTS and pykF as part of a strategy to improve SA production and its aromatic precursors in E. coli, with a resulting high yield of aromatic compounds on glucose of $0.5 \mathrm{~mol} / \mathrm{mol}$.
\end{abstract}

\section{Background}

The shikimic acid (SA) pathway is the common route leading to the biosynthesis of aromatic compounds in

*Correspondence: adelfo@ibt.unam.mx

1 Departamento de Ingeniería Celular y Biocatálisis, Instituto de Biotecnología, Universidad Nacional Autónoma de México (UNAM). Av. Universidad 2001, Col. Chamilpa, Cuernavaca, Morelos, 62210, México

Full list of author information is available at the end of the article bacteria and in several eukaryotic organisms such as ascomycetes fungi, apicomplexans, and plants [1,2]. In Escherichia coli, the first step in this pathway is the condensation of the central carbon metabolism (CCM) intermediates phosphoenol pyruvate (PEP) and erythrose 4phosphate (E4P) into 3-deoxy-D-arabinoheptulosonate 7-phosphate (DAHP) by the DAHP synthase (DAHPS) 


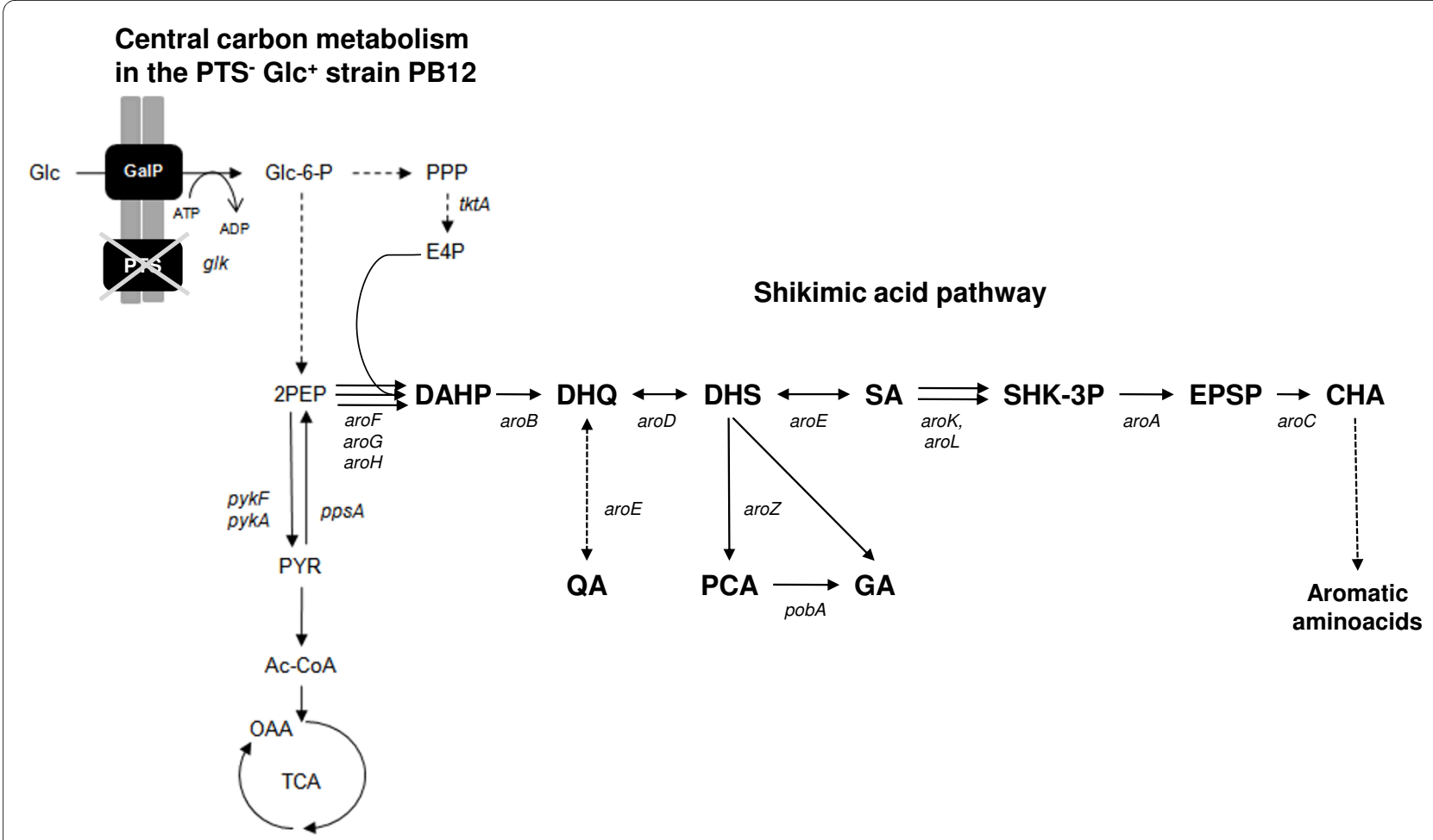

Figure 1 Central carbon metabolism and shikimic acid pathways in E. coli PB12 strain lacking the PTS. Glucose transport and phosphorylation are performed by GalP and Glk, respectively [27]. Abbreviations: Glc, glucose; GalP, galactose permease; Glc-6-P, glucose-6-P; Glk, glucokinase; PEP, phosphoenol pyruvate; PYR, pyruvate; Ac-CoA, acetyl coenzyme-A; TCA, tricarboxylic acid cycle; OAA, oxaloacetate; PPP, pentose phosphate pathway; E4P, erythrose-4-P; DAHP, 3-deoxy-D-arabinoheptulosonate-7-P; DHQ, 3-dehydroquinic acid; DHS, 3-dehydroshikimic acid; SA, shikimic acid; S3P, shikimate-3-P; EPSP, 5-enolpyruvylshikimate-3-phosphate; CHA, chorismic acid; QA, quinic acid; PCA, protocatehuic acid; GA, gallic acid. Genes and coded enzymes: $t k t A$, transketolase I;pykF, pyruvate kinase l;pykA, pyruvate kinase ll;pps $A$, phosphoenolpyruvate synthase; aroF, aroG, aroH, DAHP synthase isoenzymes $\mathrm{F}, \mathrm{G}$ and $\mathrm{H}$, respectively; $\operatorname{aroB}, \mathrm{DHQ}$ synthase; $\operatorname{aroD}, \mathrm{DHQ}$ dehydratase; aroE, shikimate dehydrogenase; aroK, shikimate kinase l; aroL, shikimate kinase II; aroA, EPSP synthase; aroC, chorismate synthase; aroZ, dehydroshikimate dehydratase; pobA, p-hydroxy-benzoate hydroxylase [32]. Continuous arrows represent unique reactions catalyzed by one or more enzymes; dotted lines or arrows represent two or more enzymatic reactions or incomplete characterized reactions.

isoenzymes AroF, AroG, and AroH, coded respectively by the aroF, aroG and $\operatorname{aroH}$ genes (Figure 1).

DAHP is converted to 3-dehydroquinate (DHQ) by dehydroquinate synthase, coded by aroB. DHQ dehydratase, coded by aroD, catalyzes the transformation of DHQ into 3-dehydroshikimic acid (DHS). This compound is reduced to SA by the shikimate dehydrogenase, coded by aroE. In turn, SA is transformed to shikimate-3P (SHK-3P) by the shikimate kinase isoenzymes I and II, coded by the aroK and aroL genes, respectively; SHK-3P is then transformed to chorismic acid (CHA) (Figure 1).

SA is used as the precursor for the synthesis of a large number of chemicals [3-5] and nowadays has gained importance as the starting compound for the chemical synthesis of the neuraminidase inhibitor oseltamivir phosphate $((3 R, 4 R, 5 S)$-4-acetylamino-5-amino-3 (1-ethylpropoxy)-1-cyclohexene-1-carboxylic acid, ethyl ester phosphate (1:1)) known as Tamiflu and produced by Roche Pharmaceuticals. This compound is currently employed as an antiviral drug for the treatment of both common seasonal influenza A and B virus infections [6,7] and for the treatment of both the avian virus type H5N1 and $\mathrm{A} / \mathrm{H} 1 \mathrm{~N} 1$ influenza infections. The latter has been considered a new pandemic $[8,9]$. It has been estimated that in the case of a global pandemic of influenza, the present capacity of Tamiflu production could be insufficient to protect large populations, particularly in developing countries $[7,8]$. Thus, alternative biotechnological strategies with engineered strains to produce SA have gained relevance.

Several metabolic engineering approaches have been developed to obtain SA from $E$. coli by biotechnological processes as an alternative to its limited and costly extraction procedures from plants such as Illicium anisatum or I. verum [3,5,9-11]. Previously developed approaches involve $E$. coli derivatives with several genetic modifications in the CCM and SA pathways. CCM modifications comprise inactivation of the PTS operon (ptsHIcrr), expression of non-PTS glucose transporters like glucose facilitators and transformation with plasmids carrying 
the $t k t A$ and $p p s A$ genes, coding for transketolase I and PEP synthase, respectively, to increase the availability of intermediates E4P and PEP, respectively [3,4,12-18]. The main modifications in the SA pathway include the partial or total blockage of the SA flux into CHA. This has been achieved by decreasing or completely eliminating the synthesis of SHK-3P -by inactivating aroK and aroL genes- with the subsequent SA accumulation (Figure 1). These modifications are commonly complemented with the transformation of plasmid-coded feedback resistant (fbr) AroF or AroG proteins (AroF ${ }^{\mathrm{fbr}}$ and AroG ${ }^{\mathrm{fbr}}$, respectively), required to avoid possible feedback inhibition in the first step of the aromatic pathway catalyzed by DAHPS isoenzymes. The rate-limiting enzyme DHQ synthase, and shikimate dehydrogenase, which is feedback inhibited by SA [3,15], catalyze two reactions that can be improved with the goal of increasing the synthesis of SA. It has been proposed that high extracellular SA concentration drives the transport of this compound into the cells by the SA transporter ShiA (shiA). Higher intracellular SA accumulation reverts the reaction catalyzed by aroE to synthesize DHS, resulting in "hydroaromatic equilibration" and by-productby formation, such as quinic (QA) and gallic acids (GA) (Figure 1). Inactivation of the ShiA transporter has been used as a strategy to reduce the intracellular accumulation of DHS, QA, and GA $[3,4,15,19,20]$. Engineered $E$. coli strains with several of the genetic modifications described above have been successfully applied to produce $71 \mathrm{~g} / \mathrm{L}$ of SA with a yield of $0.27 \mathrm{~mol} \mathrm{SA} / \mathrm{mol}$ glc and total aromatic compound yield (TACY) (including SA, DHS and QA) of $0.34 \mathrm{~mol}$ aromatic compounds/mol glc in 1-L fed-batch cultures using mineral broth with $15 \mathrm{~g} / \mathrm{L}$ yeast extract and glucose addition to maintain a 55-170 mM concentration [4]. The effects of carbon and phosphate limitations in chemostat cultures on SA production have been studied elsewhere $[15,19]$.
Our group has been involved in the characterization of E. coli strains lacking the phosphoenolpyruvate: carbohydrate phosphotransferase system (PTS-), such as strain PB12 (PTS- glc ${ }^{+}$, which has been selected as an evolved strain for growth rate recovery in a chemostat with glucose fed at progressively faster rates $[21,22]$. This strain utilizes galactose permease (GalP) and glucokinase (Glk) to transport and phosphorylate glucose into glucose-6-P, respectively (Figure 1 ). In addition, most of the glycolytic and other CCM genes are upregulated in this derivative as compared to its parental strains [21-25]. Further characterization of this evolved strain has shown increased PEP availability that can be redirected into the aromatic pathway, as compared to isogenic $\mathrm{PTS}^{+}$strains. PB12 strain has been modified for the high yield production of aromatic compounds such as L-phenylalanine $[26,27]$ and L-tyrosine [28].

In this work, we report the construction of a SA overproducing derivatives from the E. coli $\mathrm{PB} 12$ strain by inactivation of the $\operatorname{aroL}$ and $\operatorname{aroK}$ genes and expressing in plasmids different combinations of aro $G^{\mathrm{fbr}}, t k t A$, aroE, and $\operatorname{aro} B$ genes. The effects of single inactivation of either pyruvate kinase (Pyk) I or II, coded respectively by $p y k F$ and $p y k A$, were also evaluated. This strategy was used to achieve additional availability of PEP for the synthesis of aromatic compounds and SA in the E. coli PB12 PTSglc + background.

\section{Results and discussion}

Inactivation of the genes coding for shikimate kinases I and $\mathrm{II}$, and expression of the aroG $\mathrm{fbr}$, tktA, aroB and aroE genes in plasmids in the PB12 strain background

The capacity of the E. coli $\mathrm{PB} 12$ (PTS- glc ${ }^{+}$) strain to produce SA was evaluated in $500 \mathrm{~mL}$ batch cultures in $1 \mathrm{~L}$ fermentors grown in mineral broth supplemented with 25 $\mathrm{g} / \mathrm{L}$ of glucose and $15 \mathrm{~g} / \mathrm{L}$ of yeast extract. Specific growth rate $(\mu)$, glucose consumption $(q s)$, SA production and

Table 1: Growth kinetic parameters for strain PB12 and SA-producing derivatives.

\begin{tabular}{|c|c|c|}
\hline Strain/derivative & $(h-1)$ & $\begin{array}{c}\text { qsb } \\
\text { (millimol glc g DW-1 h-1)c }\end{array}$ \\
\hline $\mathrm{PB} 12$ & $0.48 \pm 0.02$ & $5.17 \times 10^{-6} \pm 4.07 \times 10^{-7}$ \\
\hline PB12.SA11 & $0.41 \pm 0.00$ & $2.03 \times 10^{-6} \pm 4.79 \times 10^{-7}$ \\
\hline PB12.SA21 & $0.42 \pm 0.02^{*}$ & $2.5 \times 10^{-6} \pm 8.55 \times 10 \times 10^{-7 *}$ \\
\hline PB12.SA22 & $0.42 \pm 0.01^{*}$ & $1.93 \times 10^{-6} \pm 5.9 \times 10 \times 10^{-7 *}$ \\
\hline PB12.SA31 & $0.32 \pm 0.02$ & $7.76 \times 10^{-6} \pm 9.67 \times 10^{-8}$ \\
\hline PB12.SA41 & $0.45 \pm 0.03$ & $2.58 \times 10^{-6} \pm 5.39 \times 10^{-7}$ \\
\hline
\end{tabular}

Values are the average of two independent experiments. a $\mu$, specific growth rate; ${ }^{b} q s$, specific glucose consumption rate; cDW, dry cell weight. Mean values within each column with the same superscript $\left(^{*}\right)(P<0.05)$ do not differ significantly with respect to the immediate parental strain (see Methods). 

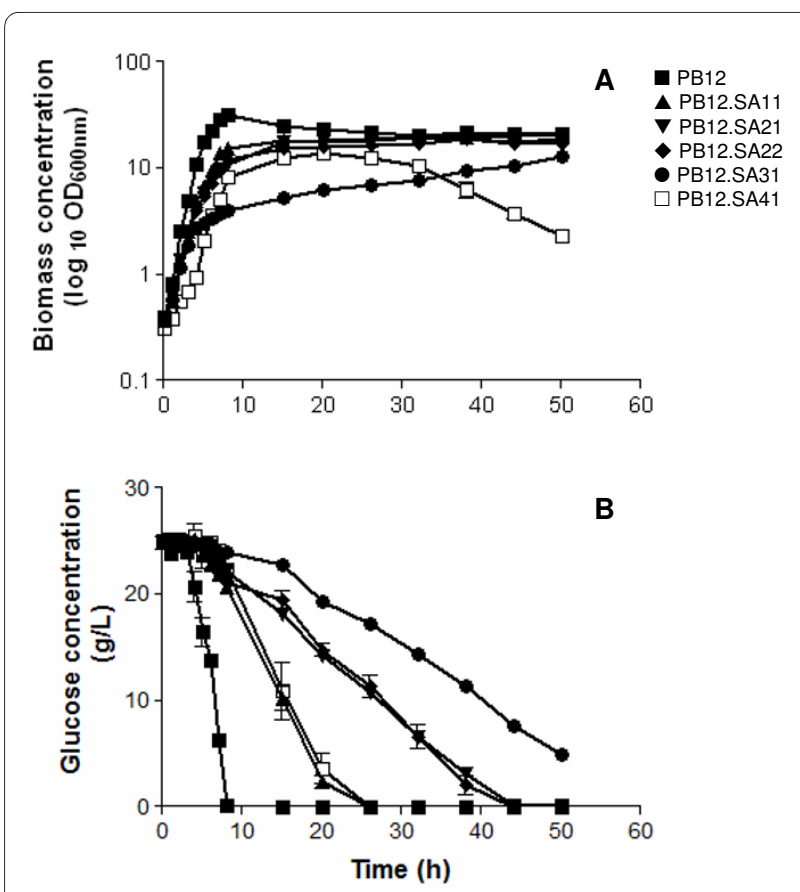

Figure 2 Biomass and glucose concentrations in PB12 and SAproducing derivatives.

yield, as well as DAHP, DHS and GA concentrations were evaluated during 50-h cultures (Table 1, Figure 2). Strain $\mathrm{PB} 12$ reached an $\mathrm{OD}_{600 \mathrm{~nm}}$ of 32, after $8 \mathrm{~h}$ of fermentation with the consumption of $98.7 \%$ of added glucose. From this time $(8 \mathrm{~h})$ to the end of the fermentation $(50 \mathrm{~h})$, a decrease in biomass concentration was observed (Figure 2). Analysis of culture supernatants showed that as expected, strain PB12 did not accumulate DAHP (Table 2, Figure 3).

Similarly to strain PB12, cultures of strain PB12.SA11 (aroL- strain expressing aroGfbr, tktA and aroB from two different plasmids) (see Methods, Figure 1 and Table 3)
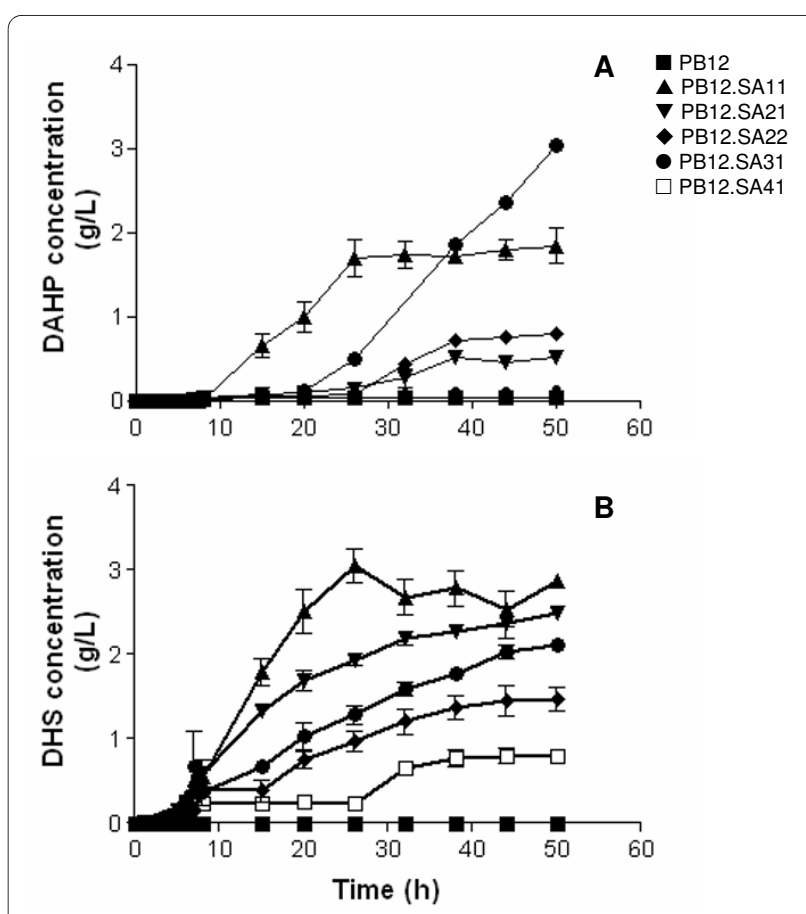

Figure 3 DAHP and DHS concentrations in PB12 and SA-producing derivatives.

also showed an exponential growth phase during the first 8-h cultivation interval as detected for strain PB12 (Figure 2). However, a significant decrease $(\mathrm{P}<0.05)$, determined by the Tukey's Honestly Significant Difference (HSD) test, was observed (see Methods) in both $\mu$ and qs values ( $86 \%$ and $39 \%$, respectively), when compared to those recorded for strain PB12 (Table 1). From this moment $(8 \mathrm{~h})$ the strain remained stationary. DAHP, DHS, SA, and GA production was detected during the exponential growth phase (Figures 3 and 4). Interestingly, relatively constant concentration levels of all aromatic intermediates were observed after glucose was com-

Table 2: Aromatic metabolites production and yields determined for strain PB12 and SA-producing derivatives.

\begin{tabular}{lcccccc}
\hline Strain & $\begin{array}{c}\text { SA } \\
\mathbf{( g / L )}\end{array}$ & $\begin{array}{c}\text { SA yield } \\
(\mathbf{m o l ~ S A / m o l} \\
\mathbf{g l c})\end{array}$ & $\begin{array}{c}\text { DAHP } \\
\mathbf{( g / L )}\end{array}$ & $\begin{array}{c}\text { DHS } \\
\text { (g/L) }\end{array}$ & $\begin{array}{c}\text { GA } \\
\text { (g/L) }\end{array}$ & $\begin{array}{c}\text { TACY1 } \\
\text { (mol aromatic } \\
\text { compounds/mol } \\
\text { glc) }\end{array}$ \\
\hline PB12 & ND & --- & $0.044 \pm 0.07$ & ND & ND & 0.00 \\
PB12.SA11 & $2.82 \pm 0.01$ & $0.11 \pm 0.00$ & $1.71 \pm 0.07$ & $2.79 \pm 0.21$ & $0.21 \pm 0.06$ & 0.28 \\
PB12.SA21 & $5.07 \pm 0.00$ & $0.21 \pm 0.00$ & $0.52 \pm 0.00$ & $2.49 \pm 0.06^{*}$ & $0.14 \pm 0.00$ & $0.33^{*}$ \\
PB12.SA22 & $7.05 \pm 0.06$ & $0.29 \pm 0.00$ & $0.81 \pm 0.04$ & $1.46 \pm 0.14$ & $0.08 \pm 0.01$ & $0.37^{*}$ \\
PB12.SA31 & $4.35 \pm 0.57$ & $0.22 \pm 0.04$ & $3.03 \pm 0.00$ & $2.12 \pm 0.02$ & $0.23 \pm 0.04$ & 0.50 \\
PB12.SA41 & $1.00 \pm 0.36$ & $0.03 \pm 0.02$ & $0.14 \pm 0.00$ & $0.79 \pm 0.01$ & ND & 0.07 \\
\hline
\end{tabular}

Values are the average of two independent experiments. 1TACY, Total aromatic compound yield (combined DAHP, DHS, SA and GA molar yields); ND, Non-detected. Mean values within each column with the same superscript $\left.{ }^{*}\right)(P<0.05)$ do not differ significantly with respect to the immediate parental strain (see Methods). 
Table 3: Strains and plasmids used and developed in this work.

\begin{tabular}{|c|c|c|}
\hline Strain/derivative & Relevant characteristics & Reference \\
\hline E. coli JM101 & $\sup E$, thi, $\triangle\left(\right.$ lac-proAB), $\mathrm{F}^{\prime}$ & {$[45]$} \\
\hline E. coli JM101 aroK- & E. coli JM101 aroK $\Delta:: \mathrm{cm}$ & This work \\
\hline E. coli aro $B^{-}$ & $\begin{array}{l}\text { E. coli K12 strain BW25113 } \triangle a r o B:: k a n \\
\text { (JW3352) }\end{array}$ & [48] \\
\hline E. coli aroE- & $\begin{array}{l}\text { E. coli K12 strain BW25113 } \triangle a r o E:: k a n \\
\text { (JW3242) }\end{array}$ & [48] \\
\hline E. coli PB28 & PB12 $\Delta p y k A:: c a t \Delta p y k F:: g e n$ & {$[31]$} \\
\hline E. coli PB12 & $\mathrm{JM} 101 \Delta(p t s \mathrm{H}-\mathrm{I}-\mathrm{crr}):: \mathrm{kan} \mathrm{glc}^{+}$ & {$[18]$} \\
\hline PB12.SA1 & PB12 $\triangle a r o L$ & This work \\
\hline PB12.SA11 & PB12.SA1 pJLBaroGfbr tktA pTOPOaroB & This work \\
\hline PB12.SA2 & PB12 $\Delta$ arol $\triangle a r o K:: c m$ & This work \\
\hline PB12.SA21 & PB12.SA2 pJLBaroGfbr tktA pTOPOaroB & This work \\
\hline PB12.SA22 & PB12.SA2 JLBaroGfbr tktA pTOPOaroB aroE & This work \\
\hline PB12.SA3 & PB12.SA2 $\Delta p y k F:: g e n$ & This work \\
\hline PB12.SA31 & PB12.SA3 pJLBaroGfbr tktA pTOPOaroB aroE & This work \\
\hline PB12.SA4 & PB12.SA2 $\Delta p y k A:: g e n$ & This work \\
\hline PB12.SA41 & PB12.SA4 pJLBaroGfbr tktA pTOPOaroB aroE & This work \\
\hline E. coli TOP10 & $\begin{array}{l}\text { F- } m c r A \Delta(m r r-h s d R M S-m c r B C) \\
\varphi 80 l a c Z \Delta M 15 \Delta l a c X 74 \text { recA1 araD139 } \\
\Delta(\text { ara-leu }) 7697 \text { galU galK rpsL endA1 nupG }\end{array}$ & Invitrogen \\
\hline \multicolumn{3}{|l|}{ Plasmids } \\
\hline pJLBaroGfbr $t k t A$ & 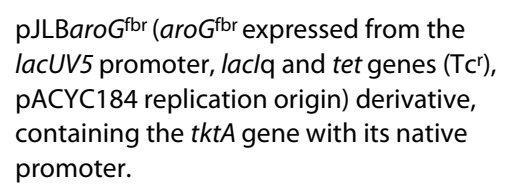 & {$[21,47]$} \\
\hline $\mathrm{pCR}^{\circ}$-Blunt II-TOPO & $\begin{array}{l}\mathrm{P}_{\mathrm{lac}} \text { lacZ- } a \text { ORF T7 promoter } c c d B \text { kan }\left(\mathrm{Km}^{\mathrm{r}}\right) \\
\text { Zeocin pUC origin. }\end{array}$ & Invitrogen \\
\hline pTOPO $\operatorname{aroB}$ & 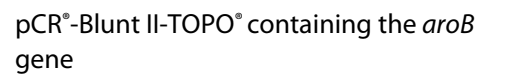 & This work \\
\hline pTOPO aroB aroE & $\begin{array}{l}\text { pTOPOaro } B \text { derivative containing the } \operatorname{aro} B \\
\text { and } \operatorname{aro} E \text { genes }\end{array}$ & This work \\
\hline
\end{tabular}

pletely consumed. DAHP, DHS, and SA accumulated during the first $26 \mathrm{~h}$ of cultivation; thereafter their concentration remained constant at around 1.71, 2.8, and $2.8 \mathrm{~g} / \mathrm{L}$, respectively (Table 2 ,). SA yield on glucose was $0.11 \mathrm{~mol} \mathrm{SA} / \mathrm{mol}$. GA concentration was lower than the other aromatic intermediates; however, as in the case of DHS, this strain produced higher GA concentrations (approximately $0.3 \mathrm{~g} / \mathrm{L}$, Table 2, Figure 4 ), than the other PB12 derivatives. It has been proposed that GA is formed by the oxidation of DHS into a diketo intermediate protocatehuic acid (PCA) followed by its spontaneous aromatization. Alternatively, this compound may result from the dehydration of DHS followed by hydroxylation of the intermediate PCA [29] (Figure 1). GA accumulation during SA production has not been reported in either batch or fed-batch cultures [4], but it has been detected in batch and chemostat cultures under carbon-limited conditions [15].

Plasmid-coded AroGfbr DAHPS avoided feedback inhibition of the first reaction of the SA pathway by the phenylalanine present in the yeast extract included in the medium or produced by the cell. It has been reported that DAHPS activity in vivo is limited by PEP and E4P availability and that maximum specific activity of DAHPS is reached when the concentration of both intermediates is increased [13,17]. It has also been reported that the presence of a plasmid-coded copy of tktA (coding for transketolase I) causes an increase in E4P availability in strain PB12 [21,22,25,30-32]. In addition, it is expected that the 

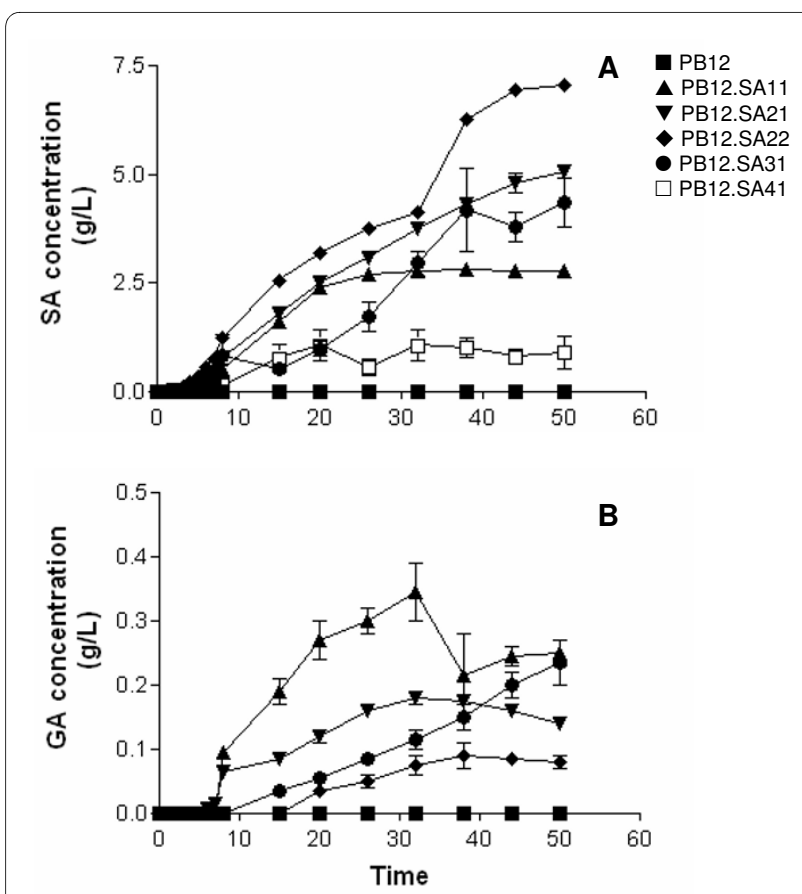

Figure 4 SA and GA concentrations in PB12 and SA-producing derivatives

presence of aroB in a multicopy plasmid (Figure 1) will reduce the possible accumulation of DAHP $[3,4]$.

Inactivation of aro $L$ gene as part of a SA production strategy has been previously described in the E. coli W3110 aroL- strain (W3110 shik1) in chemostat cultures that resulted in maximum SA yields on glucose of 0.02 and $0.05 \mathrm{~mol} / \mathrm{mol}$ under carbon and phosphate limited conditions that resulted in a maximum SA yield on glucose of $0.2 \mathrm{~mol} / \mathrm{mol}$ and $0.05 \mathrm{~mol} / \mathrm{mol}$, respectively $[15,19]$. In the present study, strain PB12.SA11 yielded $0.11 \mathrm{~mol} \mathrm{SA} / \mathrm{mol}$ glc; however, maximum yields of SA on glucose of 0.27 and $0.33 \mathrm{~mol} / \mathrm{mol}$ have been reported for another E. coli strain (PTS- $g l f$, $g l k$, aroF fbr, $t k t A$, aroE, aroK- $\left.\operatorname{aroL}^{-}\right)$in 1-L and 10-L fed-batch cultures, respectively [4], suggesting that the $\triangle a r o L$ phenotype is in itself insufficient to achieve high $S A$ yields.

Strain PB12.SA21 (aroL- aroK' strain expressing plasmid coded aro $G^{\mathrm{fbr}} t k t A$ and $\operatorname{aro} B$ genes) (Figure 1 and Table 3) showed an exponential growth phase during the first $8 \mathrm{~h}$ fermentation interval and a stationary stage similar to what was observed for PB12 and PB12.SA11 strains (Figure 2). No significant differences $(\mathrm{P}<0.05)$ were observed in $\mu$ and $q s$ values between PB12.SA21 and PB12.SA11 strains as a consequence of the inactivation of the aroL and aroK genes (Table 1). DAHP, DHS, SA, and GA production was also detected during the exponential growth phase, but important differences were observed (Figures 3 and 4). Compared to the PB12.SA11 derivative, maximum concentrations of DAHP and GA in the PB12.SA21 strain were significantly lower $(\mathrm{P}<0.05)$, whereas no significant difference $(\mathrm{P}<0.05)$ was observed in the maximum concentration of DHS (Table 2, Figure 3). Furthermore, SA production was observed throughout all the process (Figure 4). After $50 \mathrm{~h}$ of cultivation, about $5.1 \mathrm{~g} / \mathrm{L}$ of SA were detected with a yield on glucose of $0.21 \mathrm{~mol} / \mathrm{mol}$. This result represents a significant increase $(\mathrm{P}<0.05)(80 \%)$ in both SA concentration and yield, as a consequence of the double aroK- aroL- mutations (Table 2, Figure 4). The concentrations of DAHP, DHS (Figure 3) and SA obtained in strain PB12.SA11, as compared to the ones recorded for the PB12.SA21 derivative, indicate an efficient flow of aromatic intermediates from DAHP to SA. However, based on the still relatively high DHS concentration observed, it appears that this strain can further convert part of the remaining DHS to improve SA concentration and yield.

Cultures of strain PB12.SA22 (aroK aroL $^{-}$strain expressing $\operatorname{aro} G^{\mathrm{fbr}}, t k t A, \operatorname{aro} B$ and aroE from two different plasmids) (Table 3, Figure 1) showed no significant differences $(\mathrm{P}<0.05)$ in $\mu$ and $q s$ values with respect to those for strain PB12.SA21 (Table 1). Glucose was totally consumed in both strains only after $38 \mathrm{~h}$ of cultivation (Figure 2). DHS was detected in a significantly $(\mathrm{P}<0.05)$ lower concentration than the previous derivative and SA reached the highest concentration compared to all other derivatives (Table 2, Figure 4). At the end of the fermentation, $7.1 \mathrm{~g} / \mathrm{L}$ of SA were detected with a yield on glucose of $0.29 \mathrm{~mol} \mathrm{SA} / \mathrm{mol}$ (39\% increase in yield with respect to the previous derivative) and a TACY value of $0.378 \mathrm{~mol}$ aromatic compounds/mol glc (Table 2, Figure 4). Introduction of a copy of the aroE gene in the multicopy plasmid pTOPO resulted in a more efficient conversion of DHS into SA, probably as a consequence of a responsible for the synthesis of DHS from SA $[3,4,15,19,20]$. Accordingly, very small amounts of GA were produced during the cultivation of this strain (Table 2, Figure 4).

\section{Inactivation of the genes coding for pyruvate kinases I and II in the PB12SA.22 strain}

Disruption of the $p y k F$ gene in strain PB12.SA22 generated the PB12.SA31 derivative (aroL $L^{-}$, aroK $K^{-}, p y k F^{-}$strain expressing aro $G^{\mathrm{fbr}}, t k t A, \operatorname{aro} B$ and $\operatorname{aro} E$ genes from two different plasmids) (Figure 1 and Table 3). Cultures of this strain showed the characteristic two-phase growth behavior observed for the previous derivatives (Figure 2), although significant $(\mathrm{P}<0.05)$ differences were observed in $\mu$ and $q s$ values as compared to the PB12.SA22 pykF+ parental strain (Table 1). In addition, maximum biomass concentration after $8 \mathrm{~h}$ of fermentation was only 33\% with respect to the one recorded for PB12.SA22 and, contrary to all other analyzed strains, glucose was not com- 
pletely consumed after $50 \mathrm{~h}$ (Figure 2). DHS, GA, and specially DAHP final concentrations were higher than those obtained with the $p y k F^{+}$parental strain, whereas the final SA production and yield were lower (Table 2). Importantly, the TACY value in this strain was $0.50 \mathrm{~mol}$ aromatic compounds/mol glc (i.e., a 33\% increment with respect to PB12.SA22), the highest yield obtained when compared to all previous PB12.SA derivatives (Table 2).

Inactivation of the $p y k A$ gene in strain PB12.SA22 generated the derivative PB12.SA41 (aroL', aroK', pykAstrain expressing aro $G^{\mathrm{fbr}}, t k t A, \operatorname{aro} B$ and $\operatorname{aro} E$ genes from two different plasmids) (Table 3, Figure 1). Cultures of this strain showed no-significant differences $(\mathrm{P}<0.05)$ in $\mu$ and $q s$ values with respect to those recorded for the parental strain PB12.SA22 $p y k A^{+}$(Table 1). This strain reached an $\mathrm{OD}_{600 \mathrm{~nm}}$ of 14 after $20 \mathrm{~h}$ of fermentation; however, an important decrease in growth was observed from this moment to the end of the fermentation (Figure 2). This strain also showed the lowest production of DAHP, DHS, and SA as compared to all other variants, and no GA was detected (Table 2, Figures 3 and 4).

Pyruvate kinase isoenzymes Pyk I and Pyk II play a key role in the glycolytic pathway, especially in overall carbon metabolism in strains lacking PTS [33,34]. Pyk activity, together with phospho-fructokinase I and glucokinase, control the carbon flux through the glycolytic pathway and catalyze the essentially irreversible trans-phosphorylation of PEP and ADP into PYR and ATP [33]. It has been previously reported that inactivation of $p y k F$ in strain PB12 (PTS-glc $\left.{ }^{+}\right)$results in an apparently slight increase in the specific activity of Pyk A enzyme (13.5\%) [34]. Likewise, carbon flux analysis in this strain has shown a flux increase through the Pyk AF enzymes in the absence of PTS as compared to the wild-type strain (JM101 PTS+) [23]. Furthermore, transcriptome analyses in strain PB12 and in a phenylalanine overproducing PB12 derivative have shown a slight upregulation of $p y k A$ with respect to the wild type strain JM101, suggesting that the overall activity of PyK isoenzymes present in the PB12 strain is sufficient to convert PEP into PYR, at least at similar rates as in JM101 $[31,35]$. These results suggest that single inactivation of the $p y k F$ or $p y k A$ gene could be an attractive strategy to increase the amount of PEP available for DAHP synthesis, without compromising the synthesis of PYR and its flux to acetyl-CoA.

Interruption of either the $p y k F$ or $p y k A$ gene in the $E$. coli strain PB12.SA22 demonstrated a differential role of Pyk isoenzymes in overall cellular metabolism in this strain which produces aromatic compounds. Disruption of $p y k F$ in strain PB12.SA31 negatively affected growth, glucose consumption, and SA accumulation with respect to the PB12.SA22 $p y k F^{+}$parental strain. Importantly, the TACY value increased to $0.50 \mathrm{~mol}$ aromatic compounds/ mol glc in the $p y \mathrm{kF}^{-}$strain, which is $33 \%$ higher than the total yield observed for the parental strain PB12.SA22. These results suggest that $p y k F$ inactivation apparently increases PEP availability, which in turn is channeled into the aromatic pathway, resulting in a higher TACY value. Higher DAHP concentrations produced by the PB12.SA31 derivative also indicate that in this genetic background, DHQ synthase could be one of the limiting steps for SA production. This explanation contravenes the fact that this strain was transformed with a plasmidcarrying aroB; however, a previous report on the proteomic response to $p y k F$ inactivation in $E$. coli BW25113 strain demonstrated the upregulation of all the genes of the SA pathway, with the exception of $\operatorname{aro} B$, during the production of aromatic amino acids [33]. Therefore, increasing the expression of the aro $B$ gene, by substitution of its natural promoter for a stronger one, could be a viable strategy to improve SA concentrations in strain PB12.SA31.

Pyk activity plays a key role in cellular metabolism by connecting glycolysis with amino acid and lipid biosynthetic pathways [34,36,37]. Consequently, one remarkable characteristic of Pyk isoenzymes is their allosteric response to several effectors involved not only in central carbon metabolism but also in global cellular metabolism, among them, the glycolytic intermediate PEP $[34,38,39]$. It has been proposed that Pyk isoenzymes are involved in catabolite repression in E. coli glucose fermentations [40]; however, no information is available to correlate the specific role of individual Pyk isoenzymes in global bacterial metabolism, particularly in strains devoted to the production of aromatic compounds. Our results demonstrate that inactivation of the $p y k A$ gene in strain PB12.SA41 caused a negative effect on the production of aromatic compounds, probably due to an increased growth rate (Table 1). In addition, SA accumulation and TACY were substantially reduced in this strain as compared to the PB12.SA31 ( $\left.p y k F^{-}\right)$and PB12.SA22 $\left(p y k A^{+} p y k F^{+}\right)$strains. The lack of $p y k F$ clearly reduced $\mu$ and $q s$ values in relation to the parental PB12.SA22 strain. In addition, glucose was not completely consumed in strain PB12.SA31 after $50 \mathrm{~h}$, as compared to strain lacking $p y k A$, where it was completely consumed after $25 \mathrm{~h}$ (Figure 2). Furthermore, the accumulation of aromatic compounds was the highest in the strain lacking $p y k F$, while in the strain PB12.SA22, 37\% of glucose was converted into aromatic compounds; this amount increased to $50 \%$ in strain PB12.SA31. Altogether, the result of differentially inactivating the kinases I and II suggest that the PykF isoenzyme may have a more relevant role in global cellular processes than PykA in the derivatives constructed under the growth conditions tested here, since it seems that the absence of $p y k F$ apparently allows 
higher accumulation of PEP than the absence of $p y k A$. Importantly, $p y k F$ is apparently transcribed when growing on glucose from at least three different promoters in strains JM101 and PB12, while $p y k A$ is apparently only transcribed from two [41]. These results are in agreement with previous observations which suggest that PykF plays a more important role than PykA in strain JM101 (PTS ${ }^{+}$ and other derivative strains lacking PTS, when growing on glucose as the only carbon source [34].

\section{Conclusions}

E. coli $\mathrm{PB} 12\left(\mathrm{PTS}-\mathrm{glc}^{+}\right)$strain was used as the host for the synthesis of SA. The derivative PB12.SA22 was obtained by inactivation of both aroL and aroK genes, and transformed with plasmids carrying aro $G^{\mathrm{fbr}} t k t A$, aroB, and $\operatorname{aro} E$ genes. This strain was capable of efficiently channeling carbon from metabolites participating in the CCM into the aromatic pathway for the synthesis of SA. Fermentor cultures of PB12.SA22 strain in mineral broth complemented with $25 \mathrm{~g} / \mathrm{L}$ glucose and $15 \mathrm{~g} / \mathrm{L}$ yeast extract resulted in the production of $7 \mathrm{~g} / \mathrm{L}$ of SA with a yield of SA on glucose of $0.29 \mathrm{~mol} / \mathrm{mol}$ and a TACY of $0.38 \mathrm{~mol}$ aromatic compounds/mol glc. Importantly, glucose was totally consumed in strain PB12.SA22 after $48 \mathrm{~h}$ of fermentation. It is known that PTS- strains are capable of utilizing higher concentrations of glucose $(100 \mathrm{~g} / \mathrm{L})$ $[42,43]$ and different carbon sources simultaneously with glucose [29,31]. Therefore, experiments with higher glucose concentrations, including fed-batch fermentations should be performed to increase SA concentrations. In fact, preliminary results, in which glucose concentration in the medium was increased to $100 \mathrm{~g} / \mathrm{L}$, in a $500 \mathrm{~mL}$ batch fermentor cultures with strain PB12.SA22, allowed the production of $14 \mathrm{~g} / \mathrm{L}$ of SA (unpublished results).

Single inactivation of either the $p y k F$ or $p y k A$ gene was performed to further increase PEP availability for SA production in strain PB12.SA22. Inactivation of these genes demonstrated differential roles of Pyk isoenzymes in final growth, glucose consumption, and production of aromatic intermediates and SA. The $p y k F^{-}$mutation present in strain PB12.SA31 substantially affected biomass concentration, glucose consumption, and SA production, suggesting a more important role of the PykF isoenzyme in comparison to PykA, in these growing conditions. The production of SA was reduced in this strain as compared to strain PB12.SA22; however, it is notable that TACY reached a value of $0.5 \mathrm{~mol}$ aromatic compounds $/ \mathrm{mol}$ glc, which was $33 \%$ higher than the one obtained in the parental $p y k F^{+}$strain. As far as we know, there are no reports in which the utilization of a double PTS-, $p y k F^{-}$ derivative has been used to improve the production of SA and its aromatic precursors [3,4,16-18,44]. Further genetic modifications will be undertaken in this $p y k F^{-}$ derivative, such as the substitution of the $\operatorname{aro} B$ natural promoter for another that allows its upregulation to avoid the accumulation of the aromatic intermediate DAHP in order to increase the production of SA. In addition, carbon flux could still be further modulated by reducing the expression of $p y k A$ in the strain lacking $p y k F$, to obtain a higher accumulation of PEP to be channeled into the SA pathway. The $p y k A$ gene in these E. coli derivatives, as mentioned, is expressed from two different promoters when glucose is utilized as the only carbon source [41]. Therefore, it could be possible to construct derivatives lacking one of these two promoters to reduce the transcription of $p y k A$ with the goal of increasing PEP concentration.

This study demonstrated for the first time the simultaneous inactivation of PTS and $p y k F$ as part of a strategy to improve SA production and its aromatic precursors in $E$. coli, with the resulting high yield of $0.5 \mathrm{~mol}$ aromatic compounds/mol glc.

\section{Methods}

\section{Bacterial strains and plasmids}

Bacterial strains and plasmids used in this work are listed in Table 3. E. coli PB12, a derivative of strain JM101 [45], was used as the parental strain to originate the interruptions in $\operatorname{aro} L$ and $\operatorname{aroK}$ as well as the single interruption of $p y k F$ or $p y k A$. Amplification of target genes was performed with $P f u$ DNA polymerase (Fermentas, Glen Burnie, USA), according to recommendations by the supplier, in a GeneAmp PCR System thermocycler (Perkin Elmer Cetus, Norwalk, USA). Primer sets employed for amplification of target genes are listed in Table s1 (see Additional file 1). The size of the PCR products was determined by agarose gel electrophoresis. When required, amplicons were purified by cutting the desired band from the agarose gels and processed with a gel PCR purification kit (Marligen Biosciences, Urbana-PikeIjamsville, USA). The obtained derivative strains were transformed with plasmids carrying the aro $G^{\mathrm{fbr}}, t k t A$, aroB, and $\operatorname{aro} E$ genes (see below) for the construction of SA producing strains.

\section{Inactivation of the aroL gene}

PB12.SA1 strain (aroL-derivative) (Table 3) was obtained by the one-step inactivation procedure of chromosomal genes by PCR products [46]. Primer sets used are listed in Table s1 (see Additional file 1). The aroL gene was replaced by the $\Delta$ aroL:::cat cassette. Selection was performed in chloramphenicol $(\mathrm{Cm})$ containing Luria Bertani (LB) plates. Inactivation of the aroL gene in chloramphenicol resistant $\left(\mathrm{Cm}^{\mathrm{r}}\right)$ colonies was confirmed by PCR and the size of the PCR product was determined by agarose gel electrophoresis. The $\mathrm{Cm}$ cassette was deleted from the $\Delta a r o L:: c a t$ construction, as previously described [46], to facilitate subsequent gene inactivation; the $\operatorname{aroL}^{-}$genotype was confirmed by PCR. 


\section{Inactivation of the aroK gene}

Strain PB12.SA2 (aroL-, aroK- derivative) (Table 3) was constructed in a two-step procedure. First, the aroK gene of E. coli JM101 strain was replaced by the $\Delta$ aroK::cat cassette [46]. Selection was performed in $\mathrm{Cm}$ containing plates and the inactivation of $\operatorname{aroK}$ in $\mathrm{Cm}^{\mathrm{r}}$ colonies was confirmed by PCR. Second, strain PB12.SA1 was the recipient of P1 phage lysate grown on the JM101 aroK strain; the aro $K^{-}$genotype was confirmed by PCR.

\section{Inactivation of the pykF gene}

PB12.SA22 strain was the recipient of P1 phage lysate of E. coli PB28 ( $\Delta p y k F::$ gen) strain (Table 3). Transductants were selected on gentamicin $(\mathrm{Gm})$ plates and the inactivation of $p y k F$ in $\mathrm{Gm}^{\mathrm{r}}$ colonies was confirmed by PCR; the size of the PCR product was determined by agarose gel electrophoresis. The resultant strain (aroL-, aroK-, pykF-derivative) was named PB12.SA31.

\section{Inactivation of the pykA gene}

PB12.SA4 strain (aroL $L^{-}$, aroK $K^{-}$, pykA-derivative) (Table 3) was constructed by a modification of the one-step inactivation procedure of chromosomal genes by PCR products [46]. Briefly, template plasmids pKD3, pKD4, or pKD13, used to amplify FRT-resistance gene-FRT cassette, only allowed the use of $\mathrm{Cm}$ or kanamycin $(\mathrm{Km})$ as selection markers [46]; however, PB12.SA2 carried both resistance genes as a consequence of previous genetic modifications [31]. For this reason, a primer set was designed (Table s1, see Additional file 1), for priming the $\mathrm{Gm}^{\mathrm{r}}$ cassette flanked by the entire FRT sequence and homology regions for the $p y k A$ gene. The $\mathrm{Gm}^{\mathrm{r}}$ cassette was amplified using chromosomal DNA from PB12.SA3 as template and the expected product was confirmed by PCR. Purified PCR products were used to inactivate $p y k A$ in strain JM101. Selection of the resultant $\triangle p y k A:: g e n$ mutant was achieved on $\mathrm{Gm}$ containing plates and the inactivation of $p y k A$ in $\mathrm{Gm}^{\mathrm{r}}$ colonies was confirmed by PCR; the size of the PCR products was determined by agarose gel electrophoresis. The $\triangle p y k A::$ gen construction was then P1 phage transduced to PB12.SA3; $\mathrm{Gm}^{\mathrm{r}}$ colonies were selected and screened.

\section{Transformation of derivative strains with plasmid $p J L B$ aro $G^{f b r}$ tktA}

The construction of plasmid pJLB aroGfbr tktA (Table 3) has been previously reported $[21,47]$; this vector was used to transform all SA producing derivative strains. Positive clones were selected by growing colonies on LB plates supplemented with tetracycline (Tet).

\section{Cloning the aroB gene and transformation with plasmid pTOPOaroB}

The aroB gene (1484 bp) was obtained by PCR using chromosomal DNA from $E$. coli JM101 strain as template and the primers FwaroB and RvaroB (Table s1, see Additional file 1). PCR reaction was performed with $P f u$ polymerase; the size of the PCR product was determined by agarose gel electrophoresis and cloned directly into the $\mathrm{pCR}^{\circ}$-Blunt II-TOPO ${ }^{\circ}$ vector (Invitrogen, Carlsbad, USA) leading to the construction of the pTOPOaroB plasmid (Table 3). This vector was used to transform competent TOP10 cells (Invitrogen) and selection was performed on $25 \mu \mathrm{g} / \mathrm{mL}$ of zeocin-containing LB plates. Functionality of the cloned $\operatorname{aro} B$ gene was tested by restoring growth of an $\operatorname{aroB}^{-}$E. coli mutant [48] in M9 minimal medium plates supplemented with zeocin, as a consequence of the complementation of the SA pathway in this mutant strain.

\section{Cloning the aroE gene and transformation with plasmid} pTOPOaroB aroE

The aro $E$ gene ( $835 \mathrm{bp}$ ) was obtained by PCR using chromosomal DNA from E. coli JM101 strain as template and primers FwaroE and RvaroE (Table s1, see Additional file 1). PCR amplification was performed as described for the aro $B$ gene; the size of the PCR product was determined by agarose gel electrophoresis. Amplified $a r o E$ gene and plasmid pTOPOaroB were both digested with Bam HI endonuclease. This vector was treated with calf intestine phosphatase (Fermentas) and ligated with the digested aro $B$ product using T4 DNA ligase (Fermentas), transformed into TOP10 competent cells and selection was performed on zeocin-containing LB plates. Functionality of the cloned $a r o E$ gene was tested by restoring growth of an $\operatorname{aroE}^{-}$E. coli mutant [48] in M9 minimal medium plates supplemented with zeocin.

\section{Cultivation media and growth conditions}

Shake flask cultures inoculated with frozen stocks of each strain were performed in $125 \mathrm{~mL}$ baffled flasks containing $10 \mathrm{~mL}$ of LB supplemented with the respective antibiotics as required: $30 \mu \mathrm{g} / \mathrm{mL} \mathrm{Km}, 15 \mu \mathrm{g} / \mathrm{mL} \mathrm{Gm}, 20 \mu \mathrm{g} / \mathrm{mL}$ $\mathrm{Cm}$ or $30 \mu \mathrm{g} / \mathrm{mL}$ Tet (Table 3 shows specific antibiotic resistances). Cultures were incubated overnight in a shaker (New Brunswick Scientific, Edison, USA) at $37^{\circ} \mathrm{C}$, $300 \mathrm{rpm}$. An aliquot of $150 \mu \mathrm{L}$ from each culture was used to inoculate a $250 \mathrm{~mL}$ baffled flask with $50 \mathrm{~mL}$ of fermentation medium, whose composition has been previously reported for the production of $\mathrm{SA}$, and grown as described above. This medium contained $25 \mathrm{~g} / \mathrm{L}$ of glucose, $15 \mathrm{~g} / \mathrm{L}$ of yeast extract [4] and the required antibiotics. Biomass concentrations were determined and calculations were performed to adjust inoculum size to an $\mathrm{OD}_{600 \mathrm{~nm}}$ of 0.35 . Batch cultures were performed in duplicate in an Applikon autoclavable glass Bio Reactor (Schiedam, The Netherlands) $1 \mathrm{~L}$ fermentor $(500 \mathrm{~mL}$ of working volumen of fermentation medium supplemented with the required antibiotics). This device was connected to an Applikon ADI 1010 BioController and ADI 1025 controllers to monitor temperature, $\mathrm{pH}$, impeller speed and dissolved oxygen (DO). Batch fermentations were run for $50 \mathrm{~h}$ at $37^{\circ} \mathrm{C}, \mathrm{pH} 7.0$ (maintained by addition of $3.0 \% \mathrm{NH}_{4} \mathrm{OH}$ ). An impeller speed of no less than $500 \mathrm{rpm}$ 
was used to maintain DO levels at $20 \%$ air saturation. Gene expression of cloned genes was induced by adding $0.1 \mathrm{mM}$ IPTG at the onset of fermentation.

\section{Analytical procedures}

Biomass concentrations were monitored every hour during the first $8 \mathrm{~h}$ of culture; after this point they were monitored every $6 \mathrm{~h}$ until the end of the fermentation. Samples $(1.5 \mathrm{~mL})$ were withdrawn from each reactor and cell turbidity was determined spectrophotometrically at 600 nm (Beckman DU'-70 Spectrophotometer, Palo Alto, USA). Samples for the determination of SA, DHS, QA, and GA were prepared by centrifuging at $12,000 \mathrm{rpm}$ for 1 min (Eppendorff Centrifuge 5410, Brinkman Instruments Inc., Westubury, USA) $1 \mathrm{~mL}$ of fermented broth to remove cells and filtered through $0.45 \mu \mathrm{M}$ nylon membranes (Millipore, Brazil). SA, DHS, QA, and GA concentrations were determined by HPLC using a Waters system (600E quaternary pump, 717 automatic injector, 2410 refraction index, and 996 photodiode array detectors, Waters, Milford, USA), equipped with an Aminex HPX$87 \mathrm{H}$ column $(300 \times 7.8 \mathrm{~mm} ; 9 \mu \mathrm{m})$ (Bio-Rad, Hercules, USA) maintained at $50^{\circ} \mathrm{C}$. The mobile phase was $5 \mathrm{mM}$ $\mathrm{H}_{2} \mathrm{SO}_{4}$, with a flow rate of $0.5 \mathrm{~mL} / \mathrm{min}$, at $50^{\circ} \mathrm{C}$. All metabolites were detected with a photodiode array detector at $210_{\mathrm{nm}}$. DAHP concentrations were determined by the thiobarbituric acid assay [49]. This method does not distinguish between DAHP and DAH, so in this work, DAHP levels corresponded the sum of both compounds [26]. Glucose concentration was assessed by a biochemical analyzer (YSI 2700 Select, Yellow Springs, USA).

\section{Calculations}

The specific glucose $(\mathrm{S})$ consumption rate $(q s)$ was calculated during the exponential growth phase as the differential change in $\mathrm{S}$ with time $(\mathrm{t})$ normalized to the biomass concentration $\left(q s=\frac{\mu}{Y x / s}\right)$. A predetermined correlation factor $\left(1 \mathrm{OD}_{600}\right.$ corresponded to $0.37 \mathrm{~g} / \mathrm{L}$ of dry cellular weight) [50] was used to transform $\mathrm{OD}_{600}$ values into cell concentrations for $q s$ calculation. TACY determinations were based on the combined molar yields of DAHP, DHS, SA, and GA [4].

In order to determine whether the observed differences between growth, $q s$, and aromatic intermediate production (DAHP, DHS, SA and GA) in strain PB12 and in $\mathrm{PB} 12$. SA derivatives were significant $(\mathrm{P}<0.05)$, an analysis of variance (ANOVA) and the multiple comparison test of Tukey's Honestly Significant Difference (HSD) were performed using the XLSTAT program V2009.5.01 http://www.xlstat.com.

\section{Additional material}

Additional file 1 Table s1. Primers used in this work. Primers used for the amplification of inactivated and cloned genes.

Competing interests

The authors declare that they have no competing interests.

\section{Authors' contributions}

$A E$ and FB participated in the design of this study. AE and RC participated in the construction of $\triangle a r o K, \triangle A r o L, \triangle p y k F$ mutants and data analysis. RC was involved in the construction of PTOPO aroB aroE vector and fermentations. AV participated in the construction of the $\triangle p y k A$ mutant and fermentations. RA was responsible for the fermentations. GH performed HPLC determinations and data analysis. $A E, O R, G G$, and FB participated in the analysis of the results, as well as in writing and critical review of the manuscript. All authors have read and approved the manuscript.

\section{Acknowledgements}

We thank Alfredo Martínez, Shirley Ainsworth and Marcela Sánchez for critical reading of the manuscript, Mercedes Enzaldo for technical support and Paul Gaytán, Jorge Yañéz and Eugenio López for primer synthesis and DNA sequencing. This work was supported by FONSEC/SSA/IMSS/ISSSTE/CONACyT Grants 44126, 126793 and DGAPA-PAPIIT, UNAM Grants IN213508, IN224709.

\section{Author Details}

1Departamento de Ingeniería Celular y Biocatálisis, Instituto de Biotecnología Universidad Nacional Autónoma de México (UNAM). Av. Universidad 2001, Col. Chamilpa, Cuernavaca, Morelos, 62210, México and 2Departamento de Medicina Molecular y Bioprocesos, Instituto de Biotecnología, Universidad Nacional Autónoma de México (UNAM). Av. Universidad 2001, Col. Chamilpa Cuernavaca, Morelos, 62210, México

Received: 15 September 2009 Accepted: 12 April 2010 Published: 12 April 2010

\section{References}

1. Richards TA, Dacks JB, Campbell SA, Blanchard JL, Foster PG, McLeod R, Roberts CW: Evolutionary origins of the eukaryotic shikimate pathway: gene fusions, horizontal transfer, and endosymbiotic replacements. Eukaryot Cell 2006, 5:1517-1531.

2. Herrmann KM, Weaver LM: The shikimate pathway. Annu Rev Plant Physiol Plant Mol Biol 1999, 50:473-503.

3. Krämer M, Bongaerts J, Bovenberg R, Kremer S, Müller U, Orf S, Wubbolts $M$, Raeven L: Metabolic engineering for microbial production of shikimic acid. Metab Eng 2003, 5:277-283.

4. Chandran SS, Yi J, Draths KM, von Daeniken R, Weber W, Frost JW: Phosphoenolpyruvate availability and the biosynthesis of shikimic acid. Biotechnol Prog 2003, 19:808-804.

5. Adachi O, Ano Y, Toyama H, Matsushita K: High shikimate production from quinate with two enzymatic systems of acetic acid bacteria. Biosci Biothec Biochem 2006, 70:2579-2582.

6. Sheu TG, Deyde VM, Okomo-Adhiambo M, Garten RJ, Xu X, Bright RA, Butler EN, Wallis TR, Klimov Al, Gubareva LB: Surveillance for neuraminidase inhibitor resistance among human influenza $A$ and $B$ viruses circulating worldwide from 2004 to 2008. Antimicrob Agents Chemother 2008, 52:3284-3292.

7. Dharan NJ, Gubareva LV, Meyer JJ, Okomo-Adhiambo M, McClinton RC, Marshall SA, St. George K, Epperson S, Brammer L, Klimov Al, Bresee JS, Fry AM: Infections with oseltamivir-resistant influenza A (H1N1) virus in the United States. JAMA 2009, 301:1034-1041

8. Russell RJ, Lesley F, Haire LF, Stevens DJ, Collins PJ, Yi Pu Lin YP, Blackburn GM, Hay AJ, Gamblin SJ, Skehel JJ: The structure of H5N1 avian influenza 
neuraminidase suggests new opportunities for drug design. Nature 2006, 443:45-49.

9. Centers for Disease Control and Prevention: Interim guidance on antiviral recommendations for patients with confirmed or suspected swine influenza A (H1N1) virus infection and close contacts. 2009 [http://www.cdc.gov/swineflu/recommendations.htm]

10. Abbot A: What's in the medicine cabinet? Nature 2005, 435:407-409

11. Luo M: Antiviral drugs fit for a purpose. Nature 2006, 443:37-38.

12. Draths KM, Knop DR, Frost JW: Shikimic acid and quinic acid: replacing isolation from plant sources with recombinant microbial biocatalysis. J Am Chem Soc 1999, 121:1603-1604

13. LaDucca RJ, Berry A, Chotani G, Dodge TC, Gosset G, Valle F, Liao JC, YongXiao J, Power SD: Metabolic pathway engineering of aromatic compounds. In Manual of Industrial Microbiology and Biotechnology 2nd edition. Edited by: Demain AJ, Davies JE. Washington DC: ASM Press; 1999:605-615

14. Yi J, Draths KM, Li K, Frost W: Altered glucose transport and shikimate pathway product yields in E. coli. Biotechnol Prog 2003, 19:1450-1459.

15. Johansson L, Lindskog A, Silfversparre G, Cimander C, Nielsen KF, Lidén G Shikimic acid production by a modified strain of E. coli (W3110.shik1) under phosphate-limited and carbon-limited conditions. Biotechnol Bioeng 2005, 92:541-552

16. Patnaik R, Liao JC: Engineering of Escherichia coli central metabolism for aromatic metabolite production with near theoretical yield. Appl Environ Microbiol 1994, 60:3903-3908.

17. Patnaik R, Spitzer R, Liao JC: Pathway engineering for production of aromatics in Escherichia coli: Confirmation of stoichiometric analysis by independent modulation of AroG, TktA, and Pps activities. Biotechnol Bioeng 1995, 46:361-370

18. Lu JL, Liao JC: Metabolic engineering and control analysis for production of aromatics: Role of transaldolase. Biotechnol Bioeng 1997, 53:132-138

19. Johansson L, Lidén G: Transcriptome analysis of a shikimic acid producing strain of Escherichia coli W3110 grown under carbon- and phosphate-limited conditions. J Biotechnol 2006, 126:528-545.

20. Knop DR, Draths KM, Chandran SS, Barker JL, von Daeniken R, Weber W, Frost JW: Hydroaromatic equilibration during biosynthesis of shikimic acid. J Am Chem Soc 2001, 123:10173-10182.

21. Flores N, Xiao J, Berry A, Bolívar F, Valle F: Pathway engineering for the production of aromatic compounds in Escherichia coli. Nat Biotechnol 1996, 14:620-623.

22. Flores N, Leal L, Sigala JC, de Anda R, Escalante A, Martínez A, Ramírez OT, Gosset G, Bolívar F: Growth recovery on glucose under aerobic conditions of an Escherichia coli strain carrying a phosphoenolpyruvate: carbohydrate phosphotransferase system deletion by inactivating $\operatorname{arc} A$ and overexpressing the genes coding for glucokinase and galactose permease. J Mol Microbiol Biotechnol 2007, 13:105-116.

23. Gosset G, Yong-Xiao J, Berry A: A direct comparison of approaches for increasing carbon flow to aromatic biosynthesis in Escherichia coli. J Ind Microbiol 1996, 17:47-52.

24. Flores S, Gosset G, Flores N, de Graaf AA, Bolívar F: Analysis of carbon metabolism in Escherichia coli strains with an inactive phosphotransferase system by ${ }^{13} \mathrm{C}$ labeling and NMR spectroscopy. Metab Eng 2002, 4:124-137.

25. Flores N, de Anda R, Flores S, Escalante A, Hernández G, Martínez A, Ramírez OT, Gosset G, Bolívar F: Role of pyruvate oxidase in Escherichia coli strains lacking the phosphoenolpyruvate:carbohydrate phosphotransferase system. J Mol Microbiol Biotechnol 2004, 8:209-221.

26. Báez JL, Bolívar F, Gosset G: Determination of 3-deoxy-D- arabino heptulosonate 7-phosphate productivity and yield from glucose in Escherichia coli devoid of the glucose phosphotransferase transport system. Biotechnol Bioeng 2001, 73:530-535.

27. Báez-Viveros JL, Osuna J, Hernández-Chávez G, Soberón X, Bolívar F, Gosset $\mathrm{G}$ : Metabolic engineering and protein directed evolution increase the yield of L-phenylalanine synthesized from glucose in Escherichia coli. Biotechnol Bioeng 2004, 87:516-524.

28. Chávez-Béjar MI, Lara AR, López H, Hernández-Chávez G, Martinez A, Ramírez OT, Bolívar F, Gosset G: Metabolic engineering of Escherichia coli for L-tyrosine production by expression of genes coding for the chorismate mutase domain of the native chorismate mutaseprephenate dehydratase and a cyclohexadienyl dehydrogenase from Zymomonas mobilis. Appl Environ Microbiol 2008, 74:3284-3290.

29. Kambourakis S, Draths KM, Frost JW: Synthesis of gallic acid and pyrogallol from glucose: Replacing natural product isolation with microbial catalysis. J Am Chem Soc 2000, 122:9042-9043.

30. Flores S, Flores N, de Anda R, González A, Escalante A, Sigala JC, Gosset G, Bolívar F: Nutrient-scavenging stress response in an Escherichia coli strain lacking the phosphoenolpyruvate:carbohydrate phosphotransferase system, as explored by gene expression profile analysis. J Mol Microbiol Biotechnol 2005, 10:51-63.

31. Flores N, Flores S, Escalante A, de Anda R, Leal L, Malpica R, Georgellis D, Gosset G, Bolívar F: Adaptation for fast growth by differential expression of central carbon metabolism and gal regulon genes in an Escherichia coli strain lacking the phosphoenolpyruvate: carbohydrate phosphotransferase system. Metab Eng 2005, 7:70-87.

32. Martínez K, de Anda R, Hernández G, Escalante A, Gosset G, Ramírez OT, Bolívar FG: Coutilization of glucose and glycerol enhances the production of aromatic compounds in an Escherichia colistrain lacking the phosphoenolpyruvate: carbohydrate phosphotransferase system. Microb Cell Fact 2008, 7:1-12.

33. Muñoz ME, Ponce E: Pyruvate kinase: current status of regulatory and functional properties. Comp Biochem Physiol B Biochem Mol Biol 2003, 135:197-218

34. Ponce E, Flores N, Martínez A, Valle F, Bolívar F: Cloning of the two pyruvate kinase isoenzyme structural genes from Escherichia coli: the relative roles of these enzymes in pyruvate biosynthesis. J Bacteriol 1995, 177:5719-5722.

35. Báez-Viveros JL, Flores N, Juárez K, Castillo-Espana P, Bolívar F, Gosset G: Metabolic transcription analysis of engineered Escherichia coli strains that overproduce L-phenylalanine. Microb Cell Fact 2007, 6:1-20.

36. Li K, Mikola MR, Draths KM, Worden RM, Frost JW: Fed-batch fermentor synthesis of 3-Dehydroshikimic acid using recombinant Escherichia coli. Biotechnol Bioeng 1999, 64:61-73.

37. Kedar P, Colah R, Shimizu K: Proteomic investigation on the pyk- F gene knockout Escherichia coli for aromatic amino acid production. Enzyme Microb Technol 2007, 41:455-465.

38. Jurica MS, Mesecar A, Heath PJ, Shi W, NowakT, Stoddard BL: The allosteric regulation of pyruvate kinase by fructose-1,6- bisphosphate. Structure 1998, 6:195-210.

39. Fraenkel DG: Glycolysis, pentose phosphate pathway, and EntnerDuodoroff pathway. In In Escherichia coli and Salmonella typhimurium. Cellular and Molecular Biology Volume 1. Edited by: Neidhardt C. Washington DC: American Society for Microbiology; 1987:142-150

40. Drew S, Cunningham DS, Liu Z, Domagalski N, Koepsel RR, Ataai MM, Domach MM: Pyruvate kinase-deficient Escherichia coli exhibits increased plasmid copy number and cyclic AMP levels. J Bacteriol 2009, 191:3041-3049

41. Olvera L, Mendoza-Vargas A, Flores N, Olvera M, Sigala JC, Gosset G, Morett E, Bolívar F: Transcriptional analysis of central metabolism genes in Escherichia coli. Possible roles of $\sigma^{38}$ in their expression, as response to carbon limitation. PLOS ONE 2009, 4:1-23.

42. de Anda R, Lara AR, Hernández V, Hernández-Montalvo V, Gosset G, Bolivar F, Ramírez OT: Replacement of the glucose phosphotransferase transport system by galactose permease reduces acetate accumulation and improves process performance of Escherichia coli for recombinant protein production without impairment of growth rate. Metab Eng 2006, 8:281-290.

43. Lara AR, Vázquez-Limón C, Gosset G, Bolívar F, López-Munguía A, Ramírez OT: Engineering Escherichia coli to improve culture performance and reduce formation of by-products during recombinant protein production under transient intermittent anaerobic conditions. Biotechnol Bioeng 2006, 94:1164-1175.

44. Oh MK, Liao JC: Gene expression profiling by DNA microarrays and metabolic fluxes in Escherichia coli. Biotechnol Prog 2000, 16:278-286.

45. Bolívar F, Rodríguez RL, Greene PJ, Betlach MC, Heynecker HL, Boyer HW, Crosa JH, Falkow S: Construction and characterization of new cloning vehicles. II. A multipurpose cloning system. Gene 1977, 2:95-113. 
46. Datsenko KA, Wanner BL: One step inactivation of chromosomal genes in Escherichia coli K-12 using PCR products. Proc Natl Acad Sci USA 2000, 27:6640-6645.

47. Balderas-Hernández VE, Sabido-Ramos A, Silva P, Cabrera-Valladares N, Hernández-Chávez G, Báez-Viveros JL, Martínez A, Bolívar F, Gosset G: Metabolic engineering for improving anthranilate synthesis from glucose in Escherichia coli. Microb Cell Fact 2009, 8:1-12.

48. Baba T, Ara T, Hasegawa M, Takai Y, Okumura Y, Baba M, Datsenko KA, Tomita M, Wanner BL, Mori H: Construction of Escherichia coli K-12 inframe, single-gene knockout mutants: the Keio collection. Mol Syst Biol 2006, 2:1-11.

49. Weissbach A, Hurwitz J: The formation of 2-keto-3-deoxyheptonic acid in extracts of Escherichia coli B. J Biol Chem 1958, 234:705-709.

50. Hernández-Montalvo V, Martínez A, Hernández-Chávez G, Bolívar F, Valle F, Gosset G: Expression of galP and glk in a Escherichia coli PTS mutant restores glucose transport and increases glycolytic flux to fermentation products. Biotechnol Bioeng 2003, 83:687-694.

doi: $10.1186 / 1475-2859-9-21$

Cite this article as: Escalante et al., Metabolic engineering for the production of shikimic acid in an evolved Escherichia coli strain lacking the phosphoenolpyruvate: carbohydrate phosphotransferase system Microbial Cell Factories 2010, 9:21

Submit your next manuscript to BioMed Central and take full advantage of:

- Convenient online submission

- Thorough peer review

- No space constraints or color figure charges

- Immediate publication on acceptance

- Inclusion in PubMed, CAS, Scopus and Google Scholar

- Research which is freely available for redistribution

Submit your manuscript at www.biomedcentral.com/submit
C Biomed Central 